\title{
ICD-data collection features: an international survey
}

\author{
Lucia Otero Varela ${ }^{1}$, Chelsea Doktorchik ${ }^{1}$, Natalie Wiebe ${ }^{1}$, Catherine Eastwood ${ }^{1}$, Hude \\ Quan ${ }^{1}$ \\ ${ }^{1}$ Department of Community Health Sciences, Cumming School of Medicine, University of \\ Calgary
}

\begin{abstract}
The International Classification of Diseases (ICD) is globally used for coding morbidity and mortality statistics, however, its use, as well as the data collection features vary greatly across countries. To characterize hospital ICD-coded data collection worldwide. After an in-depth grey and academic literature review, an online survey was created to poll the 194 World Health Organization (WHO) member countries. Questions focused on hospital data collection systems and ICD-coded data features. The survey was distributed, using different methods, to potential participants that met the specific criteria, as well as organizations specialized in the topic, such as WHO Collaborating Centers (WHO-CC) or International Federation of Health Information Management Association (IFHIMA), to be forwarded to their representatives. Answers were analyzed using descriptive statistics. Data from 48 respondents from 26 different countries has been collected. Results reveal worldwide use of ICD, with variations in the maximum allowable coding fields for diagnoses and interventions. For instance, in some countries there is an unlimited number of coding fields (Netherlands, Thailand and Iran), as opposed to others with only 1-6 available (Guatemala or Mauritius). Disparities also exist in the definition of a main condition, as $60 \%$ of the countries use "reason for admission" and $40 \%$ utilize "resource use". Additionally, the mandatory type of data fields in the hospital morbidity database (e.g. patient demographics, admission type, discharge disposition, diagnoses, ...) differ among countries, with diagnosis timing and physician information being the least frequently required. These survey data will establish the current state of ICD use internationally, which will ultimately be valuable to the WHO for the promotion of ICD and the rollout of ICD-11. Additionally, it will improve international comparisons of health data, and encourage further research on how to improve ICD coding.
\end{abstract}

\section{Key words}

ICD, international survey, data collection, hospital database

Cite as: Otero Varela L, Doktorchik C, Wiebe N, Eastwood C, Quan H. 2019. ICD-data collection features: an international survey. Alberta Academic Review, Vol 2 (3) 2, CASCH Special Issue (not peer-reviewed), DOI: 10.29173/aar83. 